\title{
Perfil dos acadêmicos de odontologia do UNIPÊ sobre o uso de dispositivo intraoral em pacientes com reabilitação oral
}

\author{
Profile of UNIPE dentistry students on the use of intraoral device in patients with oral \\ rehabilitation
}

Perfil de estudiantes de odontología UNIPÊ sobre el uso de dispositivo intraoral en pacientes con rehabilitación oral

\author{
Vanessa Milena Rocha Silva Soares \\ ORCID: https://orcid.org/0000-0001-9077-0129 \\ Centro Universitário de João Pessoa, Brasil \\ E-mail: Vanessamilenna2@gmail.com \\ Thauany Vasconcelos Soares da Silva \\ ORCID: https://orcid.org/0000-0002-6831-9224 \\ Universidade Federal da Paraíba, Brasil \\ E-mail: vasconcelosthauany@gmail.com \\ Herrison Félix Valeriano da Silva \\ ORCID: https://orcid.org/0000-0001-6714-3151 \\ Universidade Federal da Paraíba, Brasil \\ E-mail: herrison.felix.vds@gmail.com \\ Lisandra Maria Batista Galdino \\ ORCID: https://orcid.org/0000-0003-0041-7845 \\ Centro Universitário de João Pessoa, Brasil \\ E-mail: galdinolisandra@gmail.com \\ Ana Maira Pereira Baggio \\ ORCID: https://orcid.org/0000-0002-9474-5091 \\ Universidade Estadual Paulista, Brasil \\ E-mail: anamairabaggio@gmail.com \\ Laura Gabrielle da Silva Maciel \\ ORCID: https://orcid.org/0000-0001-8348-8045 \\ Centro Universitário de João Pessoa, Brasil \\ E-mail: lauraagabrielle2@gmail.com \\ Rachel Christina de Queiroz Pinheiro \\ ORCID: https://orcid.org/0000-0002-8044-7544 \\ Centro Universitário de João Pessoa, Brasil \\ E-mail: odontogrupo@gmail.com
}

\begin{abstract}
Resumo
Este estudo tratou-se de uma pesquisa descritiva, exploratória e quantitativa que teve como finalidade analisar a percepção e o conhecimento dos estudantes de Odontologia do Centro Universitário de João Pessoa - UNIPÊ, sobre a indicação para o uso dos dispositivos interoclusais em pacientes com reabilitação oral. A amostra foi constituída por 119 estudantes do $8^{\circ}$ ao $10^{\circ}$ período do curso. O instrumento para coleta de dados consiste em um questionário o qual abordou se os alunos concordavam na relevância do uso de dispositivo interoclusal em um paciente com (DTM) e hábitos parafuncionais após reabilitação oral, e quais os benefícios. Utilizou-se a ferramenta Formulários Google da Microsoft para abordagem dos estudantes, que foram selecionados de forma não probabilística. Os dados foram tabulados em planilha EXCEL e analisados, conforme a normalidade dos dados, com auxílio de testes paramétricos ou não-paramétricos, utilizando o programa SPSS versão 20.0, considerando um nível de confiança de $95 \%$. Verificou-se também, que houve a concordância dos pesquisados que o uso do dispositivo interoclusal serve como tratamento auxiliar para as disfunções temporomandibulares $(\mathrm{n}=114 ; 95,8 \%)$, sendo necessário o uso dos dispositivos após um procedimento reabilitador em um paciente que tem ou já apresentou um quadro de disfunção temporomandibular $(n=92 ; 77,3 \%)$, de maneira complementar ao tratamento $(n=69 ; 58,0 \%)$. Conclui-se, que apesar dos resultados do estudo terem sido satisfatórios, existe uma carência na abordagem da temática no âmbito acadêmico, que por consequência resulta em um déficit nas indicações deste tratamento após a reabilitação oral em um paciente portador de DTM's.
\end{abstract}

Palavras-chave: Reabilitação bucal; Placas oclusais; Articulação temporomandibular. 


\begin{abstract}
This study was a descriptive, exploratory and quantitative research that aimed to analyze the perception and knowledge of Dentistry students at the University Center of João Pessoa - UNIPÊ, about the indication for the use of interocclusal devices in patients with oral rehabilitation. The sample consisted of 119 students from the 8th to the 10th period of the course. The instrument for data collection consists of a questionnaire which addressed whether students agreed on the relevance of using an interocclusal device in a patient with (TMD) and parafunctional habits after oral rehabilitation, and what the benefits were. Microsoft's Google Forms tool was used to approach the students, who were selected in a non-probabilistic way. Data were tabulated in an EXCEL spreadsheet and analyzed, according to data normality, with the aid of parametric or non-parametric tests, using the SPSS version 20.0 program, considering a 95\% confidence level. It was also found that there was agreement among respondents that the use of the interocclusal device serves as an auxiliary treatment for temporomandibular disorders $(n=114 ; 95.8 \%)$, requiring the use of devices after a rehabilitative procedure in a patient who has or has already presented a picture of temporomandibular disorder $(n=92 ; 77.3 \%)$, as a complement to treatment $(n=69 ; 58.0 \%)$. It is concluded that, despite the results of the study being satisfactory, there is a lack of approach to the theme in the academic context, which consequently results in a deficit in the indications for this treatment after oral rehabilitation in a patient with TMD's.
\end{abstract}

Keywords: Oral rehabilitation; Occlusal plaques; Temporomandibular joint.

\title{
Resumen
}

Este estudio fue una investigación descriptiva, exploratoria y cuantitativa que tuvo como objetivo analizar la percepción y el conocimiento de los estudiantes de Odontología del Centro Universitario João Pessoa - UNIPÊ, sobre la indicación para el uso de dispositivos interoclusales en pacientes con rehabilitación oral. La muestra estuvo formada por 119 alumnos del $8^{\circ}$ al $10^{\circ}$ período del curso. El instrumento de recogida de datos consta de un cuestionario en el que se abordaba si los estudiantes coincidían en la relevancia de utilizar un dispositivo interoclusal en un paciente con (TTM) y hábitos parafuncionales tras la rehabilitación oral, y cuáles eran los beneficios. Se utilizó la herramienta Google Forms de Microsoft para acercarse a los estudiantes, quienes fueron seleccionados de manera no probabilística. Los datos se tabularon en una hoja de cálculo EXCEL y se analizaron, según la normalidad de los datos, con la ayuda de pruebas paramétricas o no paramétricas, utilizando el programa SPSS versión 20.0, considerando un nivel de confianza del 95\%. También se encontró que hubo acuerdo entre los encuestados en que el uso del dispositivo interoclusal sirve como tratamiento auxiliar para los trastornos temporomandibulares $(n=114$; $95,8 \%$ ), requiriendo el uso de dispositivos después de un procedimiento de rehabilitación en un paciente que tiene o ha ya presentaba un cuadro de trastorno temporomandibular $(\mathrm{n}=92 ; 77,3 \%)$, como complemento al tratamiento $(\mathrm{n}=$ $69 ; 58,0 \%)$. Se concluye que, a pesar de que los resultados del estudio son satisfactorios, existe una falta de abordaje del tema en el contexto académico, lo que en consecuencia resulta en un déficit en las indicaciones de este tratamiento después de la rehabilitación oral en un paciente con TTM.

Palabras clave: Rehabilitación oral; Placas oclusales; Articulación oreja-mandíbula.

\section{Introdução}

Para Okeson (2013), as disfunções temporomandibulares (DTMs) se caracterizam por dor na região da articulação temporomandibular (ATM), músculos mastigatórios e estruturas musculoesqueléticas associadas à cabeça e ao pescoço. Pacientes com estas condições sofrem com dor, limitações funcionais da mandíbula ou estalidos na região da ATM durante qualquer movimento exercido. Um dos fatores etiológicos associados à disfunção temporomandibular (DTM) são os hábitos parafuncionais. Esse tipo de patologia está cada vez mais presente na população, devido aos estresses provindos do ritmo em que as pessoas levam a vida moderna.

Okeson (2008) enfatiza o caráter multifatorial da etiologia das DTM, no qual inúmeros fatores etiológicos podem contribuir para o aparecimento dos sinais e sintomas relacionados ao sistema estomatognático, devido à alteração da função natural deste sistema. Os fatores sistêmicos são aqueles capazes de diminuir a tolerância fisiológica do indivíduo a uma lesão ou à dor. Como exemplo, as interferências emocionais e afetivas na interpretação da dor e da disfunção, baixo condicionamento físico, má qualidade nutricional e do sono. Dentre os fatores locais estão à interferência oclusal, o traumatismo muscular ou articular e os hábitos parafuncionais.

Hábitos parafuncionais são muito comuns, e têm sido consideradas causas possíveis de (DTM). As parafunções, em contraste com os comportamentos funcionais, como mastigação, deglutição e fala, parecem não ter propósito funcional. As parafunções geralmente não causam danos ao sistema estomatognático. No entanto, quando tal atividade excede a tolerância 
fisiológica do indivíduo, ela pode causar danos à dentição, à musculatura ou à ATM, pois, os movimentos repetitivos alteram o fluxo sanguíneo normal dos tecidos musculares, ocasionando acúmulo de produtos metabólicos nas células destes tecidos, desencadeando sintomas de fadiga, dor e espasmo. A etiologia das DTM, na literatura mundial, é fundamentalmente associada ao estresse e à parafunção (Miyake et al., 2004).

Segundo Macedo et al., (2008), o uso de placas oclusais foi reportado pela primeira vez em 1901, por Karolyi et al., sendo o tratamento do bruxismo o seu principal objetivo. Com o passar do tempo, os aparelhos intraorais foram também introduzidos no tratamento das disfunções temporomandibulares (DTM) e de alguns hábitos parafuncionais. Vários tipos de placas têm sido sugeridos para esses tratamentos, destacando-se as de estabilização e as de posicionamento anterior por ser as mais utilizadas. Outros tipos de placas oclusais são: placa de mordida anterior, placa de mordida posterior, placa pivotante e placa macia ou resiliente.

Conti (2006), esclareceu que vários mecanismos têm sido sugeridos como responsáveis pela eficácia observada na maioria dos pacientes que utilizam as placas miorrelaxantes. Deve ficar claro que a utilização das placas no controle das DTMs, deve ser feita após um exame detalhado e diagnóstico correto de cada caso e, ainda, que as mesmas devem ser utilizadas em conjunto com uma série de modalidades, que incluem fisioterapia, farmacoterapia, aconselhamento e psicoterapia.

Diante disso, o objetivo deste estudo é descrever o perfil de conhecimento dos estudantes de odontologia sobre a importância para a correta indicação do uso dos dispositivos intraorais, como tratamento coadjuvante em pacientes portadores de disfunção temporomandibular e hábitos parafuncionais que tenha realizado tratamento estético reabilitador.

\section{Metodologia}

Tratou-se de uma pesquisa descritiva, exploratória e quantitativa que tem como finalidade avaliar o perfil de conhecimento dos estudantes de odontologia sobre a importância do uso de placa interoclusal em pacientes com reabilitação oral.

Neste tipo de pesquisa, os meios de coleta de dados são estruturados através de questionários de múltipla escolha, entrevistas individuais e outros recursos que tenham perguntas claras e objetivas. E estes devem ser aplicados com rigor para que se obtenha a confiabilidade necessária para os resultados. Desta forma o objetivo é medir informações sobre um assunto que já é conhecido, sendo assim os dados coletados apresentam uma natureza mais estatística e os resultados são expostos em forma de gráficos e/ou tabelas (Dalfovo, 2008).

A pesquisa seria desenvolvida nas dependências do Curso de Bacharelado em Odontologia, no Centro universitário de João Pessoa - Paraíba - Brasil, mas, em virtude das regras de distanciamento social, impostas como medida de contingenciamento à pandemia COVID19, utilizou-se através da ferramenta Formulários Google da Microsoft para abordagem dos estudantes. O link de acesso ao questionário online, assim como arquivo contendo o TCLE, foram encaminhados aos alunos, através da Coordenação do Curso, de tal forma, que não haverá acesso dos pesquisadores aos endereços eletrônicos e nomes dos mesmos. A internet facilitará o contato da pesquisadora com os participantes do estudo, além de possibilitar a disseminação do questionário de maneira rápida e segura.

A população do estudo foi constituída por acadêmicos do curso de odontologia do UNIPÊ que estavam regularmente matriculados e cursando entre o oitavo e décimo período, correspondente a 213 alunos, sendo 84 alunos do oitavo período, 65 alunos do nono período e 64 alunos do décimo período, essa é a quantidade de alunos que estavam regularmente matriculados no ano de 2020.2. Porém, desse universo de 213 alunos, apenas 119 responderam a pesquisa. Escolheu-se essa população, pelo fato deles já terem cursado as disciplinas de oclusão e prótese dentária. 


\section{Resultados e Discussão}

Os dados foram analisados por meio de estatística descritiva e testes de associação. Foram calculadas as frequências absolutas e percentuais para as variáveis categóricas. Em seguida, empregou-se o teste qui-quadrado de Pearson (ou o teste exato de Fisher quando apropriado) para determinar associação entre as variáveis. O nível de significância foi fixado em $\mathrm{p}<$ 0,05. Todas as análises foram conduzidas com o auxílio do software IBM SPSS Statistics versão 20.0, considerando um intervalo de confiança de $95 \%$.

Participaram do estudo 119 estudantes regularmente matriculados nos períodos $8^{\circ}(\mathrm{n}=37 ; 31,1 \%), 9^{\circ}(\mathrm{n}=34$; $28,6 \%)$ e $10^{\circ}(\mathrm{n}=48 ; 40,3 \%)$. A Tabela 1 mostra a distribuição dos participantes de acordo com o período e respostas às questões do questionário (parte I). Os dados evidenciaram que a maioria considera o nível de conhecimento na disciplina de Oclusão como regular, tendo aprendido o básico $(n=45 ; 37,8 \%)$, sem diferenças significativas entre os períodos $(\mathrm{p}>0,05)$. Em relação ao nível de conhecimento na disciplina de Prótese dentária, verificou-se que uma proporção maior de alunos do $8^{\circ}$ período avaliou como ótimo $(\mathrm{n}=21 ; 56,8 \%)$ em comparação com os demais, sendo a diferença estatisticamente significativa $(\mathrm{p}=0,012)$. A grande maioria relatou já ter ouvido falar em placa interoclusal $/$ placa miorrelaxante $/$ placa de bruxismo $(\mathrm{n}=$ 117; 98,3\%), principalmente em Prótese $(\mathrm{n}=89 ; 74,8 \%)$, bem como que era considerada tratamento para alguma patologia ( $\mathrm{n}$ $=92 ; 77,3 \%)$, como bruxismo $(n=73 ; 61,3 \%)$. Além disso, nestas questões não houve diferenças significativas entre os períodos $(\mathrm{p}>0,05)$.

Tabela 1. Distribuição dos participantes de acordo com o período e respostas às questões do questionário (parte I).

\begin{tabular}{|c|c|c|c|c|c|c|c|c|c|}
\hline \multirow{3}{*}{ Variáveis } & \multicolumn{6}{|c|}{ Período } & & & \\
\hline & \multicolumn{2}{|c|}{$\mathbf{8}^{\mathbf{0}}$} & \multicolumn{2}{|c|}{$9^{\circ}$} & \multicolumn{2}{|c|}{$10^{\prime}$} & \multicolumn{2}{|c|}{ Total } & \multirow[t]{2}{*}{ p-valor } \\
\hline & $\mathbf{n}$ & $\%$ & $\mathbf{n}$ & $\%$ & $\mathbf{N}$ & $\%$ & $\mathbf{n}$ & $\%$ & \\
\hline \multicolumn{9}{|c|}{ Como você considera o seu nível de conhecimento na disciplina de Oclusão? } & $0,078^{(\mathrm{b})}$ \\
\hline $\begin{array}{l}\text { Bom, sei do conteúdo, } \\
\text { mas não domino }\end{array}$ & 2 & 5,4 & 0 & 0,0 & 4 & 8,3 & 6 & 5,0 & \\
\hline $\begin{array}{l}\text { Ótimo, aprendi todo o } \\
\text { conteúdo e domino }\end{array}$ & 17 & 45,9 & 11 & 32,4 & 14 & 29,2 & 42 & 35,3 & \\
\hline $\begin{array}{l}\text { Regular, aprendi o } \\
\text { básico }\end{array}$ & 15 & 40,5 & 15 & 44,1 & 15 & 31,2 & 45 & 37,8 & \\
\hline $\begin{array}{l}\text { Péssimo, precisaria de } \\
\text { mais aulas }\end{array}$ & 3 & 8,1 & 8 & 23,5 & 15 & 31,2 & 26 & 21,8 & \\
\hline \multicolumn{9}{|c|}{ Como você considera o seu nível de conhecimento na disciplina de Prótese? } & $0,012^{(\mathbf{b}) *}$ \\
\hline $\begin{array}{l}\text { Bom, sei do conteúdo, } \\
\text { mas não domino }\end{array}$ & 7 & 18,9 & 2 & 5,9 & 7 & 14,6 & 16 & 13,4 & \\
\hline Ótimo, aprendi todo o & 21 & 56,8 & 13 & 38,2 & 18 & 37,5 & 52 & 43,7 & \\
\hline \multicolumn{10}{|l|}{ conteúdo e domino } \\
\hline $\begin{array}{l}\text { Regular, aprendi o } \\
\text { básico }\end{array}$ & 9 & 24,3 & 10 & 29,4 & 17 & 35,4 & 36 & 30,3 & \\
\hline $\begin{array}{l}\text { Péssimo, precisaria de } \\
\text { mais aulas }\end{array}$ & 0 & 0,0 & 9 & 26,5 & 6 & 12,5 & 15 & 12,6 & \\
\hline \multicolumn{9}{|c|}{ Você já ouviu falar em placa interoclusal/ placa miorrelaxante/ placa de bruxismo? } & $0,999^{\text {(b) }}$ \\
\hline $\operatorname{Sim}$ & 36 & 97,3 & 34 & 100,0 & 47 & 97,9 & 117 & 98,3 & \\
\hline Não & 1 & 2,7 & 0 & 0,0 & 1 & 2,1 & 2 & 1,7 & \\
\hline \multicolumn{10}{|c|}{ Se sim, em qual disciplina?** } \\
\hline Oclusão & 17 & 45,9 & 17 & 50,0 & 21 & 43,8 & 55 & 46,2 & \\
\hline Prótese & 27 & 73,0 & 28 & 82,4 & 34 & 70,8 & 89 & 74,8 & \\
\hline Dentística & 3 & 8,1 & 5 & 14,7 & 4 & 8,3 & 12 & 10,1 & \\
\hline Ortognática & 3 & 8,1 & 2 & 5,9 & 1 & 2,1 & 6 & 5,0 & \\
\hline
\end{tabular}


$0,243^{(a)}$

\begin{tabular}{|c|c|c|c|c|c|c|c|c|c|}
\hline \multicolumn{10}{|c|}{ Você acha que ela é tratamento para alguma patologia? } \\
\hline Sim & 25 & 67,6 & 29 & 85,3 & 38 & 79,2 & 92 & 77,3 & \\
\hline Não & 12 & 32,4 & 5 & 14,7 & 10 & 20,8 & 27 & 22,7 & \\
\hline \multicolumn{10}{|l|}{ Se sim, qual?** } \\
\hline DTM & 13 & 35,1 & 12 & 35,3 & 17 & 35,4 & 42 & 35,3 & \\
\hline Apertamento dentário & 0 & 0,0 & 6 & 17,6 & 6 & 12,5 & 12 & 10,1 & \\
\hline Bruxismo & 20 & 54,1 & 23 & 67,6 & 30 & 62,5 & 73 & 61,3 & \\
\hline Dor de cabeça & 0 & 0,0 & 0 & 0,0 & 1 & 2,1 & 1 & 0,8 & \\
\hline Dor no pescoço & 0 & 0,0 & 0 & 0,0 & 1 & 2,1 & 1 & 0,8 & \\
\hline $\begin{array}{l}\text { Dificuldade de } \\
\text { deglutição }\end{array}$ & 0 & 0,0 & 0 & 0,0 & 1 & 2,1 & 1 & 0,8 & \\
\hline Desordem oclusal & 0 & 0,0 & 0 & 0,0 & 1 & 2,1 & 1 & 0,8 & Nota. (a) \\
\hline Hábitos parafuncionais & 2 & 5,4 & 1 & 2,9 & 0 & 0,0 & 3 & 2,5 & Teste \\
\hline
\end{tabular}

quadrado de Pearson; ${ }^{(b)}$ Teste exato de Fisher; $* \mathrm{p}<0,05 ; * *$ O participante poderia assinalar mais de uma alternativa. Fonte: Autores.

De acordo com a Tabela 2, os dados mostraram que a maioria acha importante o uso da placa como tratamento auxiliar para as disfunções temporomandibulares $(\mathrm{n}=114 ; 95,8 \%)$, sendo necessário o uso de placa interoclusal após um procedimento reabilitador em um paciente que tem ou já apresentou um quadro de disfunção temporomandibular ( $\mathrm{n}=92$; $77,3 \%)$, de maneira complementar ao tratamento $(\mathrm{n}=69 ; 58,0 \%)$. Nestas questões não houve diferenças estatisticamente significativas entre os períodos (p-valores > 0,05). Em relação ao fato de saber diagnosticar um paciente que esteja precisando fazer uso da placa interoclusal, os alunos do $10^{\circ}$ período relataram saber mais $(\mathrm{n}=28 ; 58,3 \%)$ em comparação com os demais, sendo esta diferença estatisticamente significativa $(\mathrm{p}=0,044)$. Por fim, questionados sobre o que seria uma reabilitação, mais da metade dos participantes respondeu que seria um procedimento que devolve a função e estética do paciente $(\mathrm{n}=71 ; 60,2 \%)$, sem diferenças estatisticamente significativas entre os períodos $(\mathrm{p}>0,05)$.

Segundo Ommeborn et al., (2011), a placa interoclusal tem sido muito utilizada no tratamento das disfunções temporomandibulares, apertamento dental, bruxismo e no controle de hábitos parafuncionais. E de acordo com os resultados coletados da tabela 2, corroboram com a afirmação do autor, o qual os alunos da pesquisa compartilharam dessa afirmação da importância do uso da placa para tais tratamentos. Assim como, foi visto no estudo que há um consenso entre os profissionais de Odontologia que na maioria dos casos a forma de abordagem deve ser conservadora e reversível, e por isso o uso das placas interoclusais são utilizadas para esse tipo de tratamento, por apresentarem resultados positivos nos pacientes.

Tabela 2. Distribuição dos participantes de acordo com o período e respostas às questões do questionário (parte II).

\begin{tabular}{|c|c|c|c|c|c|c|c|c|c|}
\hline \multirow{3}{*}{ Variáveis } & \multicolumn{6}{|c|}{ Período } & \multirow{2}{*}{\multicolumn{2}{|c|}{ Total }} & \multirow{3}{*}{ p-valor } \\
\hline & \multicolumn{2}{|c|}{$\mathbf{8}^{\circ}$} & \multicolumn{2}{|c|}{$9^{\circ}$} & \multicolumn{2}{|c|}{$10^{c}$} & & & \\
\hline & $\mathbf{n}$ & $\%$ & $\mathbf{n}$ & $\%$ & $\mathbf{n}$ & $\%$ & $\mathbf{n}$ & $\%$ & \\
\hline \multicolumn{9}{|c|}{$\begin{array}{l}\text { Você acha que é importante o uso da placa como tratamento auxiliar para as disfunções } \\
\text { temporomandibulares? }\end{array}$} & $0,447^{(b)}$ \\
\hline $\operatorname{Sim}$ & 35 & 94,6 & 34 & 100,0 & 45 & 93,8 & 114 & 95,8 & \\
\hline Não & 2 & 5,4 & 0 & 0,0 & 3 & 6,2 & 5 & 4,2 & \\
\hline \multicolumn{9}{|c|}{$\begin{array}{l}\text { Você acha necessário o uso de placa interoclusal após um procedimento reabilitador em } \\
\text { um paciente que tem ou já apresentou um quadro de disfunção temporomandibular? }\end{array}$} & $0,243^{(a)}$ \\
\hline $\operatorname{Sim}$ & 28 & 75,7 & 23 & 67,6 & 41 & 85,4 & 92 & 77,3 & \\
\hline Não & 9 & 24,3 & 11 & 32,4 & 7 & 14,6 & 27 & 22,7 & \\
\hline \multicolumn{9}{|c|}{ Para você quão importante é o uso da placa interoclusal após uma reabilitação oral? } & $0,068^{(b)}$ \\
\hline Necessário & 12 & 32,4 & 11 & 32,4 & 26 & 54,2 & 49 & 41,2 & \\
\hline
\end{tabular}




\begin{tabular}{|c|c|c|c|c|c|c|c|c|c|}
\hline $\begin{array}{l}\text { Complementar ao } \\
\text { tratamento }\end{array}$ & 24 & 64,9 & 23 & 67,6 & 22 & 45,8 & 69 & 58,0 & \\
\hline Irrelevante & 1 & 2,7 & 0 & 0,0 & 0 & 0,0 & 1 & 0,8 & \\
\hline \multicolumn{9}{|c|}{$\begin{array}{l}\text { Você saberia diagnosticar um paciente que esteja precisando fazer uso da placa } \\
\text { interoclusal? }\end{array}$} & $0,044^{(a) *}$ \\
\hline Sim & 14 & 37,8 & 10 & 29,4 & 28 & 58,3 & 52 & 43,7 & \\
\hline Não & 23 & 62,2 & 24 & 70,6 & 20 & 41,7 & 67 & 56,3 & \\
\hline \multicolumn{9}{|c|}{$\begin{array}{l}\text { Você já tinha visto falar nesse tema "Importância do uso da placa interoclusal em } \\
\text { pacientes reabilitados"? }\end{array}$} & $0,313^{(a)}$ \\
\hline Sim & 23 & 62,2 & 15 & 44,1 & 25 & 52,1 & 63 & 52,9 & \\
\hline Não & 14 & 37,8 & 19 & 55,9 & 23 & 47,9 & 56 & 47,1 & \\
\hline \multicolumn{10}{|l|}{ Se sim, onde?** } \\
\hline Disciplina de Oclusão & 9 & 24,3 & 7 & 20,6 & 8 & 16,7 & 24 & 20,2 & \\
\hline Disciplina de Prótese & 17 & 45,9 & 14 & 41,2 & 16 & 33,3 & 47 & 39,5 & \\
\hline Exposição - trabalhos & 1 & 2,7 & 3 & 8,8 & 1 & 2,1 & 5 & 4,2 & \\
\hline Palestras & 8 & 21,6 & 7 & 20,6 & 11 & 22,9 & 26 & 21,8 & \\
\hline Minicursos & 0 & 0,0 & 0 & 0,0 & 1 & 2,1 & 1 & 0,8 & \\
\hline Instagram & 2 & 5,4 & 3 & 8,8 & 7 & 14,6 & 12 & 10,1 & \\
\hline \multicolumn{9}{|c|}{ Para você, o que é uma reabilitação oral? } & $0,287^{(\mathrm{D})}$ \\
\hline $\begin{array}{l}\text { Procedimento que } \\
\text { devolve a função e } \\
\text { estética do paciente }\end{array}$ & 24 & 64,9 & 19 & 55,9 & 28 & 59,6 & 71 & 60,2 & \\
\hline Procedimento cirúrgico & 0 & 0,0 & 1 & 2,9 & 1 & 2,1 & 2 & 1,7 & \\
\hline $\begin{array}{l}\text { Procedimento } \\
\text { estético/cirúrgico }\end{array}$ & 4 & 10,8 & 8 & 23,5 & 13 & 27,7 & 25 & 21,2 & \\
\hline $\begin{array}{l}\text { Procedimento para } \\
\text { tratamento de patologia }\end{array}$ & 9 & 24,3 & 6 & 17,6 & 5 & 10,6 & 20 & 16,9 & \\
\hline
\end{tabular}

Nota. ${ }^{(a)}$ Teste qui-quadrado de Pearson; ${ }^{(b)}$ Teste exato de Fisher; $* \mathrm{p}<0,05 ; * *$ O participante poderia assinalar mais de uma alternativa.

Fonte: Autores.

Okeson (2013), afirma que a preferência dos clínicos pela utilização das placas nos tratamentos das DTMs baseiase na observação de que as mesmas proporcionam uma terapia não invasiva, reversível e que podem apresentar excelentes benefícios na condução de diversas manifestações clínicas, assim como tratamento auxiliar dos pacientes que fazem reabilitação oral, seja por finalidade estética ou funcional, e que possuem algum tipo de DTM.

Ouso dos dispositivos intraorais, como método reversível nos tratamentos das DTM's é, sem dúvida, de extrema importância para a Odontologia, como também para tratar o paciente acometido pela mesma, devendo ser usados com prudência, tendo perícia satisfatória tanto no diagnóstico, quanto na instalação e manutenção durante todo o tratamento. Assim, o estudo de Almeida et al., (2014), corrobora com o resultado que foi analisado nos resultados da tabela 2, o qual mostra que os alunos do décimo período se mostraram mais preparados para diagnosticar a necessidade do uso da placa interoclusal.

Em um estudo realizado por Miranda (2000), conclui-se que as placas oclusais reposicionadoras são mais efetivas na redução da dor relatadas pelos pacientes, reduzindo esta sintomatologia mais rapidamente que as placas de estabilização. E, de acordo com o protocolo de utilização correto, é eficaz na redução dos danos estéticos e funcionais causados pelos hábitos parafuncionais.

Tendo em vista os resultados da pesquisa deste presente trabalho, os dados mostraram que a maioria considera importante o uso da placa como tratamento auxiliar para as disfunções temporomandibulares, mas, apenas os alunos do P10 (décimo período) se mostraram capazes de diagnosticar e compreender a importância do uso da placa após um procedimento estético e reabilitador em um paciente portador de DTM's e hábitos parafuncionais. 
Assim como no estudo de Silva et al., (2019), que foi realizado com 155 alunos do $4^{\circ}$ ao $10^{\circ}$ período foi observado que os alunos de forma geral conhecem os tipos e indicações das placas oclusais, porém os alunos do $4^{\circ}$ ao $6^{\circ}$ período apresentaram resultados mais positivos, pelo fato de estarem cursando as disciplinas de Prótese e Oclusão tornando-se assim mais familiarizados com o tema em questão.

Os resultados desse estudo e dos que foram encontrados na literatura evidenciaram que os estudantes em sua maioria dominam o conhecimento acerca da indicação do uso das placas interoclusais, e que apesar de alguns alunos não terem tido contato direto com esses dispositivos, ficou evidente que eles reconhecem a importância do uso desse tratamento nos casos de DTM's e hábitos parafuncionais e, principalmente nos pacientes que fazem tratamentos reabilitadores e padecem com algum desses distúrbios temporomandibulares. Entretanto, foi identificado que apesar dos resultados do estudo terem sido satisfatórios, existe uma carência na abordagem da temática no âmbito acadêmico, que por consequência resulta em um déficit nas indicações deste tratamento.

\section{Considerações Finais}

Os resultados obtidos neste estudo evidenciaram que os acadêmicos de Odontologia do Unipê do $8^{\circ}$ ao $10^{\circ}$ período são capazes de diagnosticar pacientes com DTM e hábitos parafuncionais que necessitam fazer uso das placas interoclusais, e com isso, conciliar com tratamento estético reabilitador. Com também, acham relevante o seu uso para melhor tempo de longevidade do tratamento estético reabilitador. Porém, apesar dos resultados terem sido satisfatórios, existe uma carência na abordagem da temática no âmbito acadêmico, que por consequência resulta em um déficit nas indicações deste tratamento como forma auxiliar nas reabilitações orais de pacientes portadores de disfunções temporomandibulares.

\section{Referências}

Almeida, C. M. et al, (2014) Dispositivos interoclusais e suas indicações no tratamento das disfunções temporomandibulares. Efdesportes.com, Revista Digital. Buenos Aires, Año 18, nº 188.

Arima, T. et al. (2012). Choice of biomaterials - Do soft occlusal splints influence jaw- muscle activity during sleep a preliminary report. Applied Surface Science, v. 262, p. 159-62.

Carrara, S. V.; ContI, P. C. R. \& Barbosa J. S. (2010). Termo do $1^{\text {o }}$ consenso em disfunção temporomandibular e dor orofacial. Dental Press J Orthod, v 15, n.3, p.114- 20 .

Conti, P. (2006). Entrevista: Paulo Conti. Rev. Dent. Press Ortodon. Ortop. Facial, v. 1, n. p. 8- 28.

Dalfovo, M. S; Lana, R. A \& SilveirA, A. (2008). Métodos quantitativos e qualitativos: um resgate teórico. Revista Interdiscīplinar Científica Aplicada, Blumenau, v.2, n.4, p.1- 13, Sem II. 2008 ISSN 1980-7031.

Gomes, C. A. F. P; Hage Y. E; Amaral A. P; Polittf \& Gonzalez D. A. B. (2014). Effects of massage therapy and occlusal splint therapy on electromyographic activity and the intensity of signs and symptoms and sleep bruxism: a randomized clinical trial. Biomed Central. v.22, n. 43, p.1-7.

Henriques, S. E. F. (1992). Efeitos de placas oclusais totais rígidas e resilientes sobre a atividade muscular noturna de pacientes com diagnóstico confirmado de parafunção. Arquivos do Centro de Estudos do Curso de Odontologia, Belo Horizonte, v. 29, n. 1, p. 35-40.

Karakis, D.; Dogan, A. \& Bek, B. (2014). Evaluation of the effect of two different occlusal splints on maximum occlusal force in patients with sleep bruxism: a pilot study. The journal of advanced prosthodontics, v. 6, n. 2, p. 103-8.

Larson, R. \& Farber, B. (2016). Estatística Aplicada. 6. ed. São Paulo: Pearson Prentice Hall.

List, T. \& Jensen R. H. (2017). Temporomandibular disorders: Old ideas and new concepts.International Headache Society. v 37 , p.7.

Miranda, M. E. \& Teixeira, M.L. (2007). A utilização das placas oclusais no controle das Disfunções Temporomandibulares (DTMs). E-book. 25 Congresso Internacional de Odontologia de São Paulo - 25 CIOSP. Cap. 8, p. 235-256.

MacedO, R. N. (2008). Bruxismo do sono. Rev. Dental Press Ortod. e Ortopedia Facial. Maringá, v. 13, n. 2, p. 18-22, 2008.

Miyake R., Ohkubo R., Takehana J. \& Morita M. (2004). Oral parafunctions and association with symptoms of temporomandibular disaorders in Japanese university students. J Oral Rehabil. v. 31, n.6, p.518- 23, 2004.

Okeson, J. P. (2008). Tratamento das desordens temporomandibulares e oclusão. Rio de Janeiro: Elsevier. 
Research, Society and Development, v. 10, n. 12, e542101220316, 2021

(CC BY 4.0) | ISSN 2525-3409 | DOI: http://dx.doi.org/10.33448/rsd-v10i12.20316

Okeson, J. P. (2013). Tratamento das desordens temporomandibulares e oclusão. 7 ed. Rio de Janeiro: Elsevier.

Oliveira, W. (2009). Disfunções temporomandibulares. São Paulo: Artes Médicas, 2002. PORTERO, P. P. et al. Placas oclusais no tratamento da disfunção temporomandibular (DTM). Revista Gestão \& Saúde, Curitiba, v. 1, n. 1, p. 36-40.

Ommerborn, M. A. (2011). Therapies most frequently used for the management of bruxism by a sample of German dentists. The Journal of Prosthetic Dentistry, St. Louis, v. 105, n. 3, p.194-202,2011.

Portero, P.P. et al. Placas oclusais no tratamento da disfunção temporomandibular (DTM). Revista Gestão\&Saúde, Curitiba, v.1, n.1, p. 36-40. 2009. http://www.herrero.com.br/files/revista/fileb5e3973b7f4f21920c93addd6d1853fb.pdf Acesso em: 12 nov. de 2019.

Reis, M. Q. (2015). Método de confecção de placa oclusal: Ajuste simplificado. Belo Horizonte Faculdade de odontologia Universidade Federal de Minas Gerais.

Silva. E. V. F et al. (2018). Reabilitação estética e funcional em paciente com desgaste oclusal dental acentuado: Relato de caso clínico. Revista Odontológica de Araçatuba, v.39, n.1, p. 21-7.

Silva, K. R. N. et al. (2017). Análise do grau de conhecimento dos estudantes de odontologia de uma instituição de ensino particular de Teresina-PI sobre placas. In: Anais da Mostra de Pesquisa em Ciência e Tecnologia. 